\section{BMJ}

Open

Gastroenterology

\title{
Validation of Novel Fibrosis Index (NFI) for assessment of liver fibrosis: comparison with transient elastography (FibroScan)
}

\author{
Azhar Hussain (D) , ${ }^{1}$ Muhammad Asif Gul, ${ }^{2}$ Muhammad Usama Khalid (D) ${ }^{1}$
}

To cite: Hussain A, Gul MA, Khalid MU. Validation of Novel Fibrosis Index (NFI) for assessment of liver fibrosis: comparison with transient elastography (FibroScan). BMJ Open Gastro 2019;6:e000316. doi:10.1136/ bmjgast-2019-000316

Received 28 May 2019 Revised 26 June 2019 Accepted 4 July 2019
Check for updates

\section{(C) Author(s) (or their} employer(s)) 2019. Re-use permitted under CC BY-NC. No commercial re-use. See rights and permissions. Published by BMJ.

${ }^{1}$ Ameer-ud-Din Medical College, PGMI, Lahore, Pakistan ${ }^{2}$ Gastroenterology, Nishtar Medical College and Hospital, Multan, Pakistan

Correspondence to Mr Azhar Hussain; azharnewton0786@gmail.com

\begin{abstract}
Background In this study, we collated cheap and readily available non-invasive biomarkers and FibroScan score in predicting fibrosis stages in chronic hepatitis $\mathrm{C}$ virus $(\mathrm{HCV})$ infection.

Methods We studied 1898 patients with HCV infection confirmed by presence of HCV RNA in their serum. We compared the FibroScan score and fibrosis indices (FIs): aspartate transaminase (AST) to alanine transaminase (ALT) ratio (AAR), AST to Platelet Ratio Index (APRI), FI, fibrosis-4 (FIB-4), Age-Platelet Index (API), Pohl score, Fibrosis Cirrhosis Index (FCl). We developed a new FI, named Novel Fibrosis Index (NFI) calculated by the following formula: $\mathrm{NFI}=\left[\left(\right.\right.$ bilirubin $\left.\times(\mathrm{ALP})^{2}\right) /($ platelet count (albumin) $\left.\left.)^{2}\right)\right]-n$.

Results AAR, APRI, FI, FIB-4, API, Pohl score, FCl and $\mathrm{NFI}$ were able to predict fibrosis stage with correlation coefficient indices $0.848,0.711,0.618,0.741,0.529$, $0.360,0.477$ and 0.26 , respectively. Receiver operating characteristic curves showed sensitivity and specificity for predicting $\mathrm{F} 3$ by $\mathrm{NFI}=75.1 \%$ and $41.1 \%$ and $\mathrm{F} 4$ for $\mathrm{NFI}=72.1 \%$ and $47.1 \%, \mathrm{AAR}=62.8 \%$ and $37.6 \%$, APRI $=74.6 \%$ and $87.6 \%$, FIB- $4=53.2 \%$ and $72.3 \%$, $\mathrm{Fl}=78.1 \%$ and $92.3 \%, \mathrm{APl}=78.1 \%$ and $60 \%$, Pohl score $=38.1 \%$ and $78.1 \%$ and $\mathrm{FCl}=78.1 \%$ and $88.1 \%$. Conclusions Our NFI predicted F3 and has been found to have more sensitivity and specificity in predicting F4 fibrosis stage than other Fls.
\end{abstract}

\section{INTRODUCTION}

Chronic viral hepatitis $\mathrm{C}$ virus (HCV) infection has more prevalence in low-income and middle-income countries with low to limited national per capita income. ${ }^{1}$ According to WHO report 2017, Egypt has highest prevalence and Pakistan has the second highest prevalence. ${ }^{2}$ According to a survey conducted in 2018, 18 million Pakistanis are infected with hepatitis B virus (HBV) and $\mathrm{HCV}$ which is about $9 \%$ of country's population and the number of people losing life to hepatitis every day in Pakistan is 400 .

Most commonly we encounter $\mathrm{HCV}^{3}$ which, in most cases, progresses to chronic hepatitis disease. The progression can be slow or rapid,

\section{Summary box}

What is already known about this subject?

- FibroScan is replacing invasive liver biopsy.

- Cost and availability of FibroScan facility has been a major issue for low-income and middle-income countries.

What are the new findings?

- We successfully evaluated almost all the readily available biomarkers in one study.

- We developed another serum index named Novel Fibrosis Index (NFI) which can predict both F3 and F4 stages.

How might it impact on clinical practice in the foreseeable future?

- Easy to follow-up the progression of fibrosis with no repercussions and minimum inconvenience to patient and will reduce work burden in healthcare departments as compared with other methods.

- Obesity that constraints the diagnostic yield of FibroScan has no inference on diagnostic yield of our NFI.

- Most cost-effective and easy approach among FibroScan and other NITs with comparable diagnostic accuracy.

- Our NFI has been found to have more sensitivity and specificity in predicting F4 fibrosis stage than any other fibrosis serum index available at present. It also predicted F3 (advanced fibrosis stage) with considerable sensitivity and specificity and this stage is not predicted by any other fibrosis serum index available at present.

consistent or inconsistent depending on the degree of active tissue inflammation and damage ${ }^{4}$; it usually progresses gradually over many years. ${ }^{3}$ HCV does not cause cell death directly but provokes immune inflammatory response such as chronic injury to hepatocytes causing release of interleukin-2 and various other cytokines to stimulate Ito cells and fibroblasts to start synthesising collagen type I, especially that result in progressive fibrosis, hepatocellular damage and cell death. 
Chronic viral infection causes multiple waves of inflammation and tissue repair which involves deposition of extracellular matrix resulting in scarring or progressive fibrosis over time and ultimately leading to liver cirrhosis. ${ }^{5}$ Cirrhosis eventually causes onset of multiple decompensating events leading to decompensated liver disease. ${ }^{6}$ Decompensated liver disease or decompensated cirrhosis is the state where liver is not able to perform all of its functions reasonably. Actually, liver failure, in most cases, develops gradually over the years.

Stages of liver fibrosis in chronic HCV infection is a well-established factor that determines the severity of disease and associated complications, that is, hepatic encephalopathy, ascites, portal hypertension, etc, which are associated with F4 stage of fibrosis mostly. Therefore, the definite assessment of stages of liver fibrosis in chronic HCV infection is required to determine urgency of treatment and therapy outcome. Moreover, the precise evaluation of fibrosis is quite essential in indicating when to begin antiviral therapy in patients with chronic HCV infection, thereby completely eradicating virus in each case in low-income and middle-income countries. ${ }^{7}$

Identification of infected individuals early in the disease process is the crucial point of management and follow-up. It is the assessment of progression to cirrhosis that is of high priority in devising treatment plan of each patient. This assessment remains a substantial challenge. ${ }^{8}$

Conventionally, liver biopsy had been a gold standard for staging of fibrosis, ${ }^{9}$ however, it is an invasive procedure requiring workforce (skilful handling), bringing discomfort and significant expense to patient. Moreover, internal bleeding may occur thereafter. Variability among observers ${ }^{10}$ in scoring liver biopsies, inability to follow-up progression and a sampling error up to $30 \%$ is also problematic. ${ }^{11}$

A number of non-invasive markers have been developed which are useful supplements to asses stages of fibrosis. These are biomarkers (aspartate transaminase (AST) to alanine transaminase (ALT) ratio (AAR), AST to Platelet Ratio Index (APRI), fibrosis index (FI), fibrosis-4 (FIB-4), Age-Platelet Index (API), Pohl score, Fibrosis Cirrhosis Index (FCI)) and radiological markers including MRI and transient elastography. ${ }^{12}$

Ultrasound-based FibroScan is one recent development (first machine inaugurated in 2010 in Pakistan at the National Institute of Liver and Gastrointestinal Diseases, Dow University), but it is quite expensive technique and its universality or accessibility is also an issue in curbing prevalent hepatitis in low-income and middle-income countries. Moreover, obesity is another limitation in diagnostic yield of FibroScan. ${ }^{13}$

We tried to examine whether the combination of certain biomarkers can make assessment of liver fibrosis more precise. Then, we tried to evaluate and asses the combined diagnostic presentation of cheap and easily accessible biomarkers if they could serve same purpose as FibroScan score in predicting prognosis of chronic liver disease in clinical practice.

\section{A priori hypothesis}

Validation of cheap and readily available serum biomarkers in correlation to transient elastography and developing a new index, Novel Fibrosis Index (NFI), if that can give prognosis of liver fibrosis without essential reliance on FibroScan that may not be available or unaffordable to patient.

\section{MATERIALS AND METHODS}

This study was carried out at Hepatitis Clinic, Lahore General Hospital/Ameer-ud-Din Medical College, Lahore, Pakistan. We explained the whole process of our study to the patients. Informed consent was obtained from patients who were willing to be involved in research. It was a cross-sectional study. This study was carried out from 11 February 2017 to 29 December 2018. Study was approved by ethical review board.

Patients with chronic HCV infection were identified among the patients visiting Hepatitis Clinic, Lahore General Hospital, Lahore, who were only positive for hepatocellular carcinoma (HCC) antibodies by detecting HCV RNA by PCR and then, HCV genotype was established. $\mathrm{HBV} / \mathrm{HCV}$ and $\mathrm{HCV} / \mathrm{HIV}$ co-infected patients and on which any clinical findings of liver cancer were present, were not included in the study. Total 1898 patients were engaged over this period. Quantitative determination of the FibroScan score (Liver Stiffness Index), baseline viral load obtained by PCR and biomarkers (liver function tests (LFTs), albumin, bilirubin and complete blood count (CBC)) were done. The fibrosis stages of patients were determined from FibroScan score using Metavir System. We considered results of FibroScan reliable if $\mathrm{IQR} /$ median value was $<30 \%$. We took consecutive 10 readings of FibroScan and considered average of these readings as our FibroScan score value. Then we used Ziol transient elastography breaking points for staging of fibrosis according to Metavir System of fibrosis: 2.5-8.8 FibroScan value was labelled as F0-F1, 8.9-9.6 FibroScan value as F2, 9.7-14.6 FibroScan value as F3 and $>14.6$ labelled as F4. The patients were assessed for readily available serum FIs: AAR, APRI, FI, FIB-4, API, Pohl score, FCI and our newly developed NFI.

The following formulas were used to review the predicted scores with particular cut-off values:

- AAR=AST $(\mathrm{IU} / \mathrm{L}) / \mathrm{ALT}(\mathrm{IU} / \mathrm{L})$

If AST $/$ ALT $\geq 1$, significant cirrhosis.

- APRI=[\{AST (IU/L)/ALT_ULN $(\mathrm{IU} / \mathrm{L})\} \times 100] /$ platelet count $(109 / \mathrm{L})$

If $A P R I<0.5$, no or minimal fibrosis; if APRI $>1.5$, significant fibrosis.

- FI=8.0-0.01×PLT $(109 / \mathrm{L})$-serum albumin $(\mathrm{g} / \mathrm{dL})$

If $\mathrm{FI}<2.1$, no or minimal fibrosis; $\mathrm{FI} \geq 2.1$, significant fibrosis and $\mathrm{FI} \geq 3.3$, significant cirrhosis.

- FIB-4=[age $($ years $) \times$ AST $(\mathrm{IU} / \mathrm{L})] /[$ platelet count $(\times 109 / \mathrm{L}) \times$ ALT $(\mathrm{IU} / \mathrm{L}) 1 / 2]$

If FIB- $4<1.45$, no or minimal fibrosis, if FIB- $4>3.25$, significant fibrosis. 
- FCI $=($ alkaline phosphatase $\times$ serum bilirubin/serum albumin $\times$ platelet count)

If $\mathrm{FCI}<0.131$, significant fibrosis; if $\mathrm{FCI}>1.25$, significant cirrhosis.

- API=age/Platelet Index

Age $\quad($ years $)<30=0 ; \quad 30-39=1 ; \quad 40-49=2 ; \quad 50-59=3$; $60-69=4 ; \geq 70=5$.

Platelet count $\left(10^{9} / \mathrm{L}\right): \geq 225=0 ; 200-224=1 ; 175-199=2$; $150-174=3 ; 125-149=4 ;<125=5$.

It ranges from 0 to 10 , where $0-2=$ no or minimal fibrosis, $3-5=$ mild fibrosis with few septa formation and $6 \geq$ bridging fibrosis to cirrhosis and/or moderate-to-severe necroinflammatory lesions.

- Pohl score; AST:ALT:platelet count $\left(10^{9} / \mathrm{L}\right)$

If AST $/ \mathrm{ALT}<1$ and platelet count $>150000$ then excludes marked fibrosis.

\section{Calculation and development of NFI}

We also developed a new index for predicting stages of fibrosis naming it as NFI. Our newly developed NFI was developed by observing the various relationships and variations of serum bilirubin, alkaline phosphatase, platelet count and serum albumin in liver fibrosis caused by chronic HCV infection by continuously noticing the routinely used and cost-effective tests of patients suffering from chronic HCV infections including LFTs and CBC. Keeping in mind these observations and variations in patients with chronic HCV infection, we put values of serum bilirubin, alkaline phosphatase, platelet count and serum albumin as variables in Microsoft Excel sheet to formulate an equation for NFI. We did not use gamma GT, collagen III and V and hyaluronic acid as variables in our study because they are not used routinely in hepatic clinics of many low-income countries and are not cost-effective for the patients and if we used gamma GT, collagen III and V and hyaluronic acid as variables in our study, they will render our newly developed index non-affordable and poorly reproducible for the poor patients who are supposed to carry the heavy burden of direct-acting antiviral medications as well:

$$
\mathrm{NFI}=\left[\frac{\text { Bilirubin } \times(\text { ALP })^{2}}{\text { Platelet Count }(\text { Albumin })^{2}}\right]-\mathbf{n}
$$

Where $n=2000$ and ' $n$ ' is constant that is introduced to accommodate measurement in small values which is more convenient to use.

Then, we applied various biostatistical tests such as independent sample T-test, linear curve estimation analysis, Spearman's rank correlation and Pearson's rank correlation coefficients to observe NFI's relationship with FibroScan test to determine FibroScan score. After observing linear relationship, we drew receiver operating characteristic (ROC) curves to calculate cut-off value, sensitivity and specificity of NFI in predicting F3 and F4 stages of fibrosis in chronic HCV infection.

\section{Statistical analysis}

The data were analysed using statistical package SPSS Windows V.22. We considered $p$ value $<0.05$ as statistically significant. To determine the significant association between continuous variables and liver fibrosis stages, Spearman's rank correlation was used. The Student's t-test was used to collate arithmetic means and parameters while $\chi^{2}$ test was used to collate categorical data. The univariate and multiple regression analyses were done for different biomarkers. ROC curves were performed and area under the ROC curves were used to collate and infer the diagnostic accuracies of the serum fibrosis indexes along with their cut-off points, sensitivities and specificities.

\section{RESULTS}

We studied on 1898 patients: 1124 (59.2\%) were females, $774(40.7 \%)$ were males. One thousand seven hundred sixty-seven $(93.1 \%)$ were married and $131(6.9 \%)$ were unmarried. The number of labourers were 1066 (56.2\%), housewives were $719(37.9 \%)$ and female government employees were 113 (6\%).

Distribution of different fibrosis stages among our sample population were $\mathrm{F} 0-\mathrm{F} 1=1034(54.5 \%), \mathrm{F} 2=112$ $(5.9 \%), \mathrm{F} 3=253(13.3 \%)$ and $\mathrm{F} 4=499(26.3 \%)$. Majority of patients $(1235,65.1 \%)$ had viral genotype $3 a$, patients having genotype $1 \mathrm{~b}$ were $581(30.6 \%)$ and patients having la genotype were $82(4.3 \%)$.

\section{Descriptive statistics}

Descriptive statistics is presented in table 1.

The independent sample T-test results for stage F0-F1 and F2 and F3 and F4 for different variables with FibroScan score determined fibrosis stage.

The independent sample T-test results also indicated statistically significant relationship $(\mathrm{p}<0.05)$ of all of our variables with Fibroscan score.

The relationship of AAR, APRI, FI, FIB-4, FFCI, API, Pohl Score and NFI with FibroScan score in univariate analysis was found to be statistically significant $(\mathrm{p}<0.05)$ with $\mathrm{R}^{2}$ value of $0.848,0.711,0.618,0.741,0.529,0.360$ and 0.477 , respectively.

Linear curve estimation analysis with analysis of variances for albumin, bilirubin, platelet count, ALT, AST, alkaline phosphatase, AAR, APRI, FI, FIB-4, FCI and NFI showed a statistically significant relationship with Pearson's correlation coefficient $(r)$ values: $0.451(p<0.05)$, $0.336(\mathrm{p}<0.05), 0.597(\mathrm{p}<0.05), 0.100(\mathrm{p}<0.05), 0.087$ $(\mathrm{p}<0.05), 0.100(\mathrm{p}<0.05), 0.087(\mathrm{p}<0.05), 0.492(\mathrm{p}<0.05)$, $0.091(\mathrm{p}<0.05), 0.334(\mathrm{p}<0.05), 0.568(\mathrm{p}<0.05)$ and 0.455 $(\mathrm{p}<0.05)$, respectively.

\section{ROC curve analysis}

ROC curve analysis for validation of serum AAR, APRI, FIB-4, FI, API, Pohl score and FCI were performed and sensitivity and specificity along with cut-off points (table 2) were calculated for F0-F3 and F4 (figures 1 and 2). We put F0-F3 together in a single group because majority of population remains asymptomatic during these stages of fibrosis in chronic HCV infection for years mostly, which is followed by F4 (cirrhotic stage) and its 
Table 1 Descriptive statistics

\begin{tabular}{|c|c|c|c|c|c|}
\hline & $\mathbf{N}$ & Minimum & Maximum & Mean & SD \\
\hline Age of patient (years) & 1898 & 14.0 & 100.0 & 41.550 & 12.8913 \\
\hline Baseline viral load & 1898 & 119 & 107911144 & 1101257.94 & 6075925.912 \\
\hline Albumin (g/dL) & 1898 & 1.70 & 11.00 & 3.4440 & 1.26541 \\
\hline ALT (IU/mL) & 1898 & 8.0 & 7000.0 & 78.195 & 171.6666 \\
\hline AST (IU/mL) & 1898 & 14.0 & 1085.0 & 74.195 & 64.9119 \\
\hline Bilirubin $(\mathrm{mg} / \mathrm{dL})$ & 1898 & 0.20 & 24.00 & 1.1946 & 1.22437 \\
\hline FibroScan score & 1898 & 2.60 & 76.00 & 13.3569 & 13.00018 \\
\hline AAR & 1898 & 0.05 & 9.94 & 1.0697 & 0.54862 \\
\hline Platelet count $\left(100 \mathrm{000} / \mathrm{mm}^{3}\right)$ & 1898 & 17.90 & 26800.00 & 502.3153 & 2299.09585 \\
\hline APRI & 1898 & 0.00 & 8.24 & 0.8769 & 1.03031 \\
\hline $\mathrm{FI}$ & 1898 & -262.72 & 6.20 & -0.3871 & 22.94007 \\
\hline FIB-4 & 1898 & 0.00 & 23.28 & 1.7149 & 1.88862 \\
\hline $\mathrm{FCl}$ & 1898 & 0.00 & 47.72 & 0.7808 & 1.98782 \\
\hline Alkaline phosphatase (IU/L) & 1898 & 51.0 & 1154.0 & 301.946 & 137.8091 \\
\hline API & 1898 & 0.0 & 10.0 & 2.893 & 2.8627 \\
\hline Pohl score & 1898 & 0.0 & 1.0 & 0.663 & 0.4729 \\
\hline NFI & 1898 & -3.20 & 233629.57 & 7498.4298 & 14670.79515 \\
\hline
\end{tabular}

AAR, AST to ALT ratio; ALT, alanine aminotransferase; API, Age-Platelet Index; APRI, AST to Platelet Ratio Index; AST, aspartate aminotransferase; FCl, Fibrosis Cirrhosis Index; FI, Fibrosis Index; FIB-4, fibrosis-4; NFI, Novel Fibrosis Index.

associated complications, that is, hepatic encephalopathy, ascites, portal hypertension, etc.

\section{PREDICTABILITY OF NFI}

Results of ROC curve analysis for our newly developed NFI for F3 and F4 with appropriate cut-off values and their sensitivities and specificities are presented in table 3 and ROC curves are shown in figures 3 and 4. Comparison of different biomarkers of study with NFI is shown in comparative ROC curves in figures 5 and 6.

\section{Our newly developed NFI}

Our NFI is very useful for predicting F3 and F4 stages of fibrosis in chronic HCV infection and is reliable because its findings coincide with FibroScan test predicted fibrosis stages, that is, F3 and F4 and also are supported strongly by clinical findings in patients with chronic HCV infection.

\section{DISCUSSION}

Hepatic cirrhosis caused by chronic HCV infection and non-alcoholic fatty liver disease are leading contributors in deaths caused by chronic diseases. Cirrhosis does not develop simultaneously but it takes a mean infection time of approximately 30 years and it may occur in different ages with different age ranges, that is, $10-50$ years. Fibrosis in connective tissue followed by its extension in hepatic tissue in HCV infection is an evidence of cirrhosis. ${ }^{14} 15$

Genotype 3a was the most prevalent genotype found in our study and our results are reinforcing the already existing studies on prevalence of various genotypes of HCV virus in Pakistan. ${ }^{14} 15$ There were large number of patients with $\mathrm{F} 0-\mathrm{F} 1$, that is, none or initial fibrosis stage which is followed by cirrhosis (F4). Mean age $>40$ years was found to be significantly related to marked fibrosis and cirrhosis and our study augmented the results of other studies as well.

Liver biopsy has been advised as a gold standard to evaluate the fibrosis stage, yet invasive, costly and serious repercussions such as soreness, bile leakage, haemorrhages, infection, severe right hypochondriac pain, lacerations and other severe complications can lead to death, with $1.6 \%$ mortality rate noted in a study.15 Liver biopsy also requires expert hands for sampling and is not cost-effective for the patients. ${ }^{16}$ Various researches narrated the host factors contemplating the fibrosis development that can ultimately lead to HCC. ${ }^{12}$ Their usage is compatible as non-invasive means to get rid of the drawbacks of invasive biopsy. ${ }^{17} \mathrm{HCV}$ infection is linked with abnormally high levels of aminotransferases in blood staying $>6$ months. Stage of liver fibrosis determines the basis on which different treatment regimens are planned.

Previously many studies tried to find out the authentic, non-distressing biomarkers and tried to determine the ties between aminotransferases level, hyaluronic acid levels, number of platelets, collagen levels and baseline viral load with fibrosis but to no avail, as results were uncertain. Since then different thresholds of several scoring indices like AAR, APRI, FI, FIB-4, API, Pohl score and FCI have been proclaimed to anticipate the existence and non-existence of fibrosis or cirrhosis in patients infected with HCV. However, earlier stages of fibrosis and mild fibrosis cannot be determined accurately by using 
Table 2 ROC curve analysis for validation of serum AAR, APRI, FIB-4, FI, API, Pohl score and FCl for F3 and F4 in 1898 patients with HCV infection

\begin{tabular}{|cllll|}
\hline Stage & $\begin{array}{l}\text { Cut-off } \\
\text { value }\end{array}$ & $\begin{array}{l}\text { Specificity Sensitivity } \\
(\%)\end{array}$ & AUC \\
\hline AAR & & & & \\
\hline F0-F3 & $<1$ & 41.9 & 62.5 & 0.377 \\
\hline F4 & $>1$ & 37.6 & 62.8 & 0.412 \\
\hline APRI & & & & \\
\hline F0-F3 & $<0.5$ & 68.0 & 56.2 & 0.54 \\
\hline F4 & $>1.5$ & 87.6 & 74.8 & 0.864 \\
\hline FIB-4 & & & & \\
\hline F0-F3 & $<1.45$ & 65.4 & 51 & 0.521 \\
\hline F4 & $>3.25$ & 72.3 & 53.2 & 0.801 \\
\hline
\end{tabular}

\section{$\mathrm{Fl}$}

\begin{tabular}{cllll} 
F0-F3 & $<2.1$ & 34.4 & 82.2 & 0.556 \\
\hline F4 & $>3.3$ & 92.3 & 78.1 & 0.826 \\
API & & & & \\
F0-F3 & $<2.5$ & 58.4 & 70 & 0.624 \\
F4 & $>2.5$ & 60 & 78.1 & 0.578
\end{tabular}

\section{Pohl score}

\begin{tabular}{cllll} 
F0-F3 & 0 & 58.4 & 30 & 0.499 \\
F4 & 1 & 78.1 & 38.1 & 0.599 \\
FCl & & & & \\
F0-F3 & $<0.131$ & 57.4 & 37 & 0.499 \\
F4 & $>1.25$ & 88.1 & 78.1 & 0.867 \\
\hline
\end{tabular}

AAR, AST to ALT ratio; ALT, alanine transaminase; API, AgePlatelet Index; APRI, AST to Platelet Ratio Index; AST, aspartate transaminase; AUC, area under the curve; $\mathrm{FCl}$, Fibrosis Cirrhosis Index; FI, Fibrosis Index; FIB-4, fibrosis-4; HCV, hepatitis $\mathrm{C}$ virus; ROC curve, receiver operating characteristic curve.

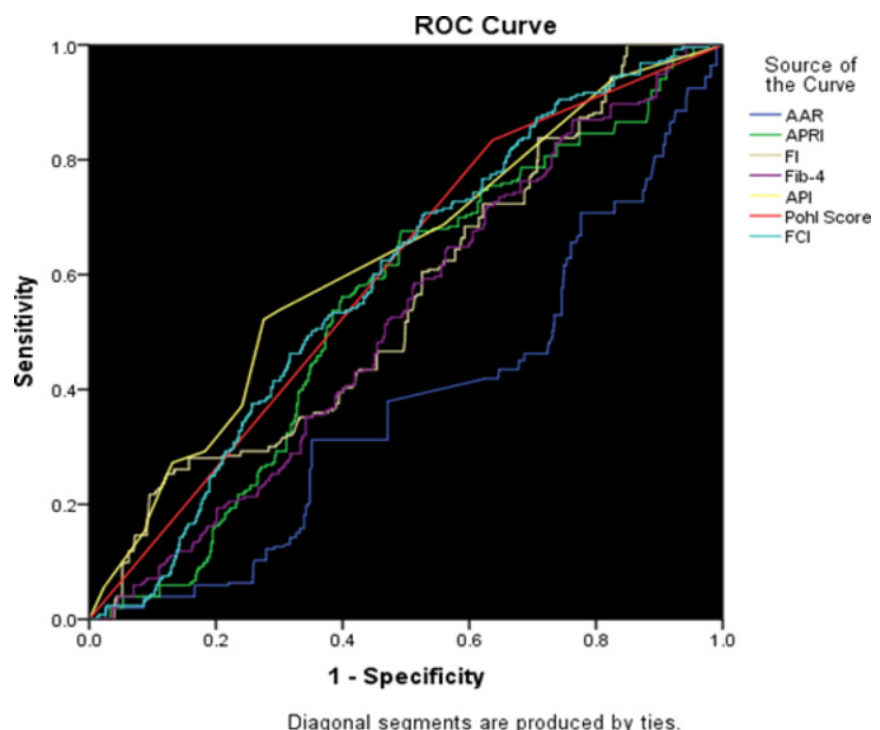

Figure 1 Receiver operating characteristic (ROC) curves of different biomarkers for F0-F3. AAR, AST to ALT ratio; ALT, alanine transaminase; API, Age-PlateletIndex; APRI, AST to Platelet Ratio Index; AST, aspartate transaminase; $\mathrm{FCl}$, Fibrosis Cirrhosis Index; FI, Fibrosis Index; FIB-4, fibrosis-4.

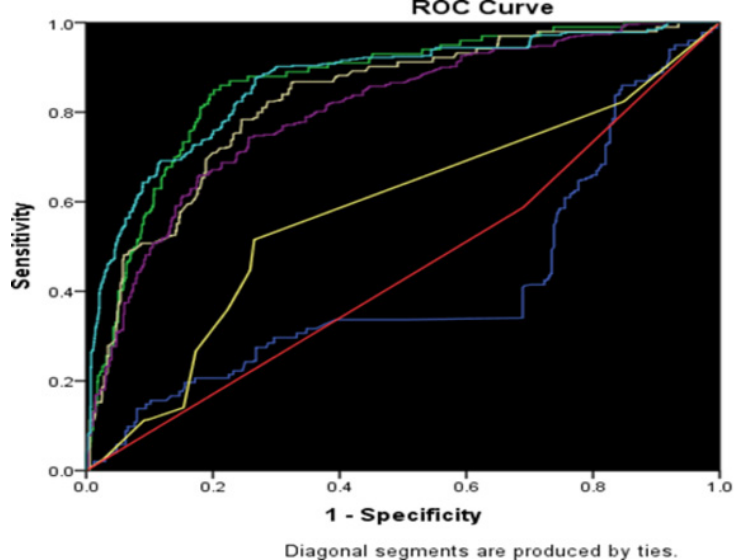

Source of
the Curve - AAR 二AARI

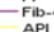
- Pohl Score

Figure 2 Receiver operating characteristic (ROC) curves of different biomarkers for F4. AAR, AST to ALT ratio; ALT, alanine transaminase; API, Age-Platelet Index; APRI, AST to Platelet Ratio Index; AST, aspartate transaminase; $\mathrm{FCl}$, Fibrosis Cirrhosis Index; FI, Fibrosis Index; FIB-4, fibrosis-4.

only one structure of biomarkers, also all the readily available indices have some limitations like inability to differentiate all fibrosis stages singly and some have been constructed primarily for patients having co-infection. ${ }^{18}$ In this study, we collated the diagnostic performance of non-invasive indices with transient elastography, that is, FibroScan, which are available to predict cirrhosis like AAR, APRI, FI, FIB-4, API, Pohl score and FCI. They are cheap, readily available, non-invasive and cost-effective than other non-invasive techniques like transient elastography, that is, FibroScan. ${ }^{19}$ For this objective and assessment of much earlier stages of fibrosis, we refined a new serum fibrosis index by evaluating several clinical and pathological aspects.

Our results back the latest recommendations by the European Association for the Study of the Liver to apply non-invasive tests as first-line tests in prognostication of liver fibrosis. ${ }^{20-22}$ According to our conclusions and these new recommendations, liver biopsy is needed only if redundant non-invasive tests show dissension. Blood markers can be used to predict cirrhosis and advanced stages of fibrosis and should be used if transient elastography is not available or cost-effective to patient or when diagnostic yield is constrained as in patients with obesity. $^{23}$

For AAR at cut-off value $<1$, sensitivity was $62.5 \%$ and specificity was $41.9 \%$ and area under curve (AUC) was

Table 3 ROC curve analysis for validation of NFI for F3 and F4 in 1898 patients with HCV infection

\begin{tabular}{lllll} 
& \multicolumn{3}{l}{ NFI } & \\
Stage & Cut-off value & $\begin{array}{l}\text { Specificity } \\
\text { (\%) }\end{array}$ & $\begin{array}{l}\text { Sensitivity } \\
\text { (\%) }\end{array}$ & AUC \\
\hline F3 & $>11.64$ & 75.1 & 61.1 & 0.609 \\
F4 & $>30.94$ & 72.1 & 47.1 & 0.831 \\
\hline
\end{tabular}

AUC, area under the curve; HCV, hepatitis C virus; NFI, Novel Fibrosis Index; ROC curve, receiver operating characteristic curve. 


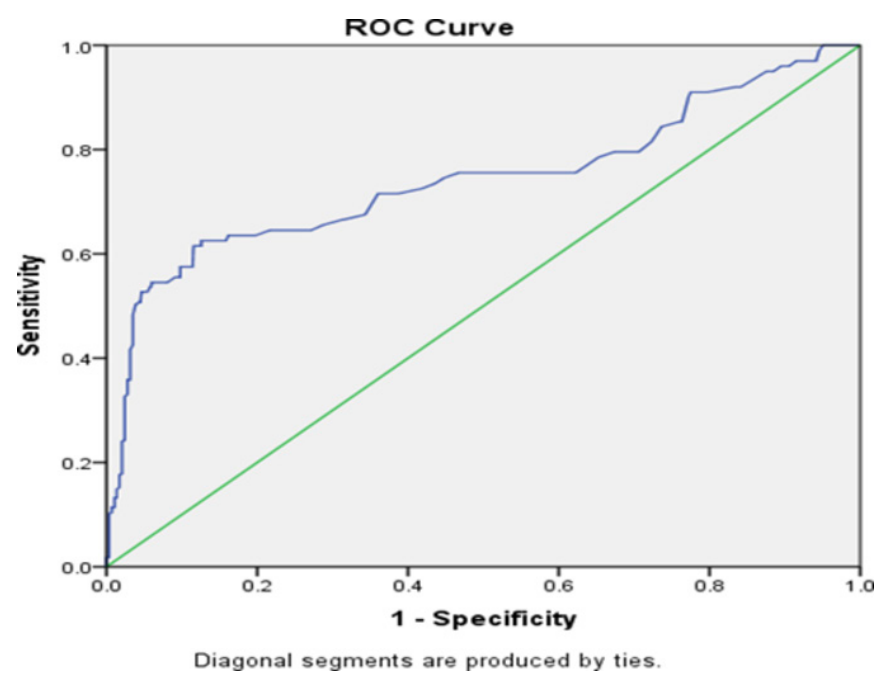

Figure 3 Receiver operating characteristic (ROC) curve of Novel Fibrosis Index for F3.

0.377 for predicting F0-F3. At cut-off value $>1$, sensitivity and specificity were $62.8 \%$ and $37.6 \%$, respectively, with $\mathrm{AUC}=0.412$ for $\mathrm{F} 4$ and our study results support the results of other studies. ${ }^{24}$

For APRI at cut-off value $<0.5$, sensitivity and specificity for predicting $\mathrm{F} 0-\mathrm{F} 3$ were $56.2 \%$ and $68.0 \%$, respectively, with $\mathrm{AUC}=0.546$. At cut-off value $>1.5$, sensitivity and specificity were $74.6 \%$ and $87.6 \%$, respectively, with $\mathrm{AUC}=0.864$ for $\mathrm{F} 4 \ldots{ }^{25}$

FIB-4 was invented by Afify et al in $2006 .{ }^{26}$ At cut-off value $<1.45$, sensitivity and specificity for predicting F0F3 were $51 \%$ and $65.4 \%$, respectively and AUC was 0.521 . At cut-off value $>3.25$, sensitivity and specificity were $53.2 \%$ and $72.3 \%$, respectively, with $\mathrm{AUC}=0.801$ for $\mathrm{F} 4$ and our study findings support the results of Afify et al, which concluded the same sensitivity and specificity for predicting F0-F3 and F4 as well for FIB- $4 .^{27}$

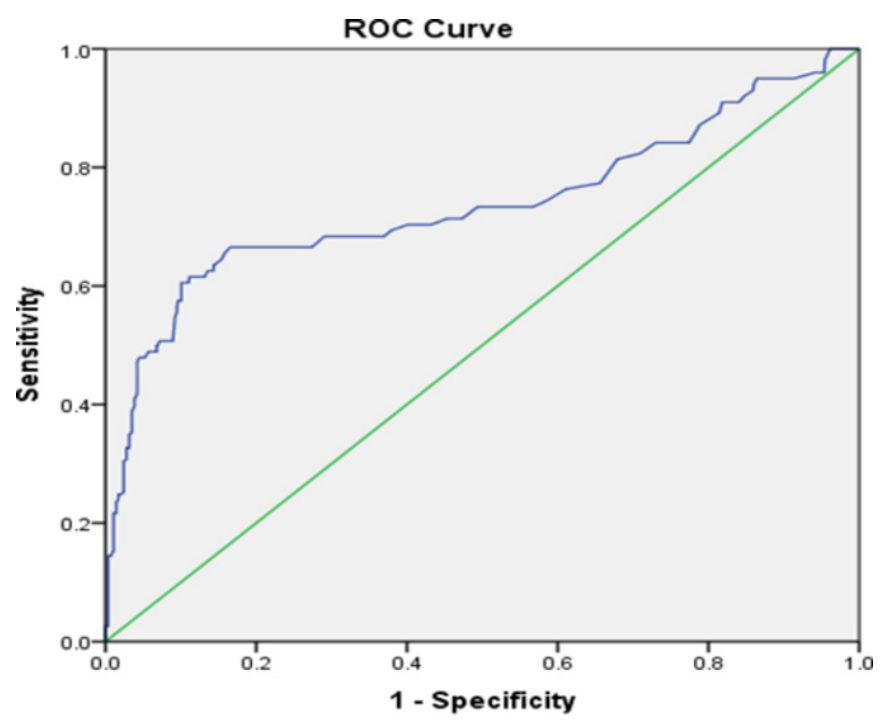

Diagonal segments are produced by ties.

Figure 4 Receiver operating characteristic (ROC) curve of Novel Fibrosis Index for F4.

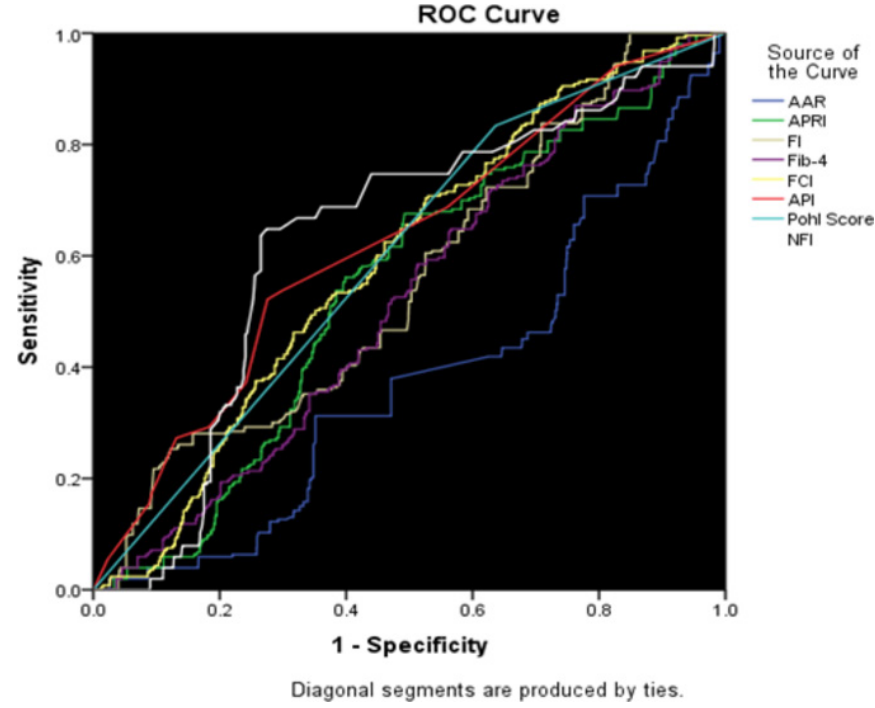

Figure 5 Comparitive receiver operating characteristic (ROC) curve of NFI with different biomarkers for F3. AAR, AST to ALT ratio; ALT, alanine transaminase; API, AgePlateletIndex; APRI, AST to Platelet Ratio Index; AST, aspartate transaminase; $\mathrm{FCl}$, Fibrosis Cirrhosis Index; FI, Fibrosis Index; FIB-4, fibrosis-4; NFI, Novel Fibrosis Index.

Like other studies on non-invasive biomarkers, ${ }^{19}{ }^{24} \mathrm{FI}$ at cut-off value $<2.1$, sensitivity and specificity for predicting F0-F3 were $82.2 \%$ and $34.4 \%$, respectivelyand AUC was 0.556 . At cut-off value $>3.3$, sensitivity and specificity were $78.1 \%$ and $92.3 \%$, respectively, with $\mathrm{AUC}=0.826$ for predicting F4.

For API at cut-off value $<2.5$, sensitivity and specificity for predicting $\mathrm{F} 0-\mathrm{F} 3$ were $70 \%$ and $58.4 \%$, respectively and AUC was 0.624 . At cut-off value $>2.5$, sensitivity and specificity were $78.1 \%$ and $60 \%$, respectively, with $\mathrm{AUC}=0.578$ for predicting $\mathrm{F} 4$ and study findings coincided with other studies as well.

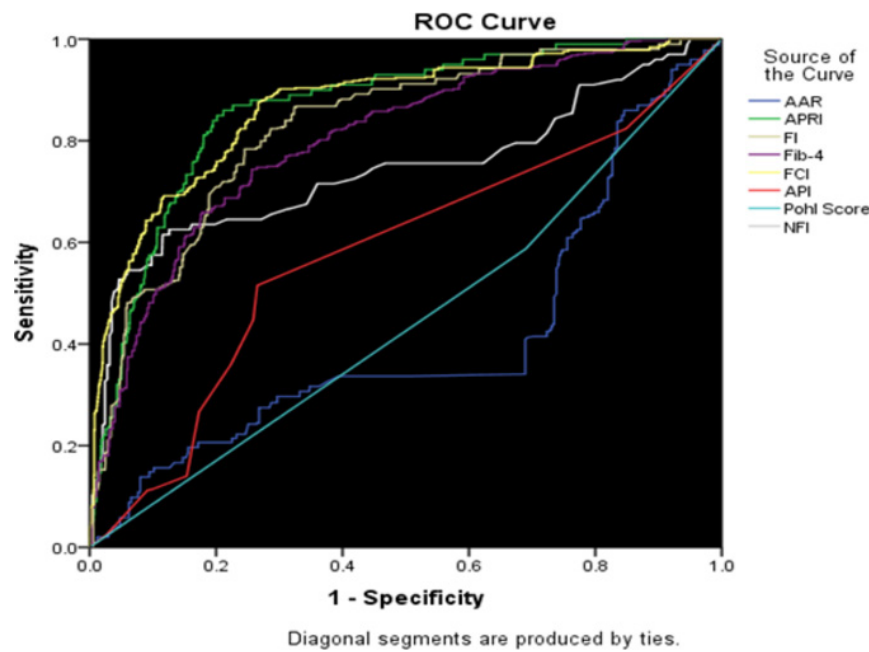

Figure 6 Comparitive receiver operating characteristic (ROC) curve of NFI with different biomarkers for F4. AAR, AST to ALT ratio; ALT, alanine transaminase; API, AgePlatelet Index; APRI, AST to Platelet Ratio Index; AST, aspartate transaminase; $\mathrm{FCl}$, Fibrosis Cirrhosis Index; FI, Fibrosis Index; FIB-4, fibrosis-4; NFI, Novel Fibrosis Index. 
Pohl score was not found to be a good index to stage fibrosis. At cut-off value $<0$, sensitivity and specificity for predicting F0-F3 were $30 \%$ and $58.4 \%$, respectively and AUC was 0.499 . At cut-off value $>1$, sensitivity and specificity were $38.1 \%$ and $78.1 \%$, respectively, with $\mathrm{AUC}=0.549$ for $\mathrm{F} 4$.

Ahmed $e t a l$ studied the same relationship of FCI and fibrosis stages and FCI, in our study like previous one, was found to be a good test in predicting cirrhosis than non-cirrhotic stages. At cut-off value $<0.131$, sensitivity and specificity for predicting F3 were 37\% and 57.4\%, respectively and AUC was 0.529 . At cut-off value $>1.25$, sensitivity and specificity were $78.1 \%$ and $88.1 \%$, respectively with $\mathrm{AUC}=0.867$ for $\mathrm{F} 4$.

At $>11.64$, NFI had specificity and sensitivity of $75.1 \%$ and $61.1 \%$, respectively, with $\mathrm{AUC}=0.609$ for predicting F3. At $>30.94$, NFI had specificity and sensitivity of $72.1 \%$ and $47.1 \%$, respectively, with $\mathrm{AUC}=0.831$ for predicting F4. So, our NFI has been found to be highly efficient in staging fibrosis than any other fibrosis serum index available at present.

\section{CONCLUSIONS}

Our NFI predicted F3 and has been found to have more sensitivity and specificity in predicting $\mathrm{F} 4$ fibrosis stage than other FIs.

Contributors AH designed the study; collected, arranged data and contributed in statistical analysis of data and writing manuscript. MUK collected lab work's data, compiled it, helped in designing the research, most importantly contributed in writing paper and critically analysing it. All work was performed under supervision of MAG including data collection. He also read manuscript critically and played a vital role in designing project. All authors approved the final manuscript.

Funding The authors have not declared a specific grant for this research from any funding agency in the public, commercial or not-for-profit sectors.

Competing interests None declared.

Patient consent for publication Not required.

Ethics approval This study was approved by Institutional Ethical Review Board, Lahore General Hospital, Lahore, Pakistan.

Provenance and peer review Not commissioned; externally peer reviewed.

Data availability statement Data are available on reasonable request. All data relevant to the study are included in the article or uploaded as supplementary information.

Open access This is an open access article distributed in accordance with the Creative Commons Attribution Non Commercial (CC BY-NC 4.0) license, which permits others to distribute, remix, adapt, build upon this work non-commercially, and license their derivative works on different terms, provided the original work is properly cited, appropriate credit is given, any changes made indicated, and the use is non-commercial. See: http://creativecommons.org/licenses/by-nc/4.0/.

\section{ORCID iDs}

Azhar Hussain http://orcid.org/0000-0002-2935-6954

Muhammad Usama Khalid http://orcid.org/0000-0002-0498-2430

\section{REFERENCES}

1. Liaw Y-F. Antiviral therapy of chronic hepatitis B: opportunities and challenges in Asia. J Hepatol 2009;51:403-10.

2. World Health Organization. Pakistan tackles high rates of hepatitis from many angles, 2017. Available: http://www.who.int/news- room/ featurestories/detail Pakisan-tackles-high-rates-of-hepatitis-frommany-angles [Accessed at 29 November, 2018].

3. Vegnente A, Larcher VF, Mowat AP, et al. Duration of chronic active hepatitis and the development of cirrhosis. Arch Dis Child 1984;59:330-5.

4. Tsochatzis EA, Bosch J, Burroughs AK. Liver cirrhosis. The Lancet 2014;383:1749-61.

5. Iredale JP. Models of liver fibrosis: exploring the dynamic nature of inflammation and repair in a solid organ. $J$ Clin Invest 2007; 117:539-48.

6. Keltch B, Lin Y, Bayrak C. Comparison of Al techniques for prediction of liver fibrosis in hepatitis patients. J Med Syst 2014;38:60.

7. Marcellin P, Gane E, Buti M, et al. Regression of cirrhosis during treatment with tenofovir disoproxil fumarate for chronic hepatitis $\mathrm{B}$ : a 5-year open-label follow-up study. The Lancet 2013;381:468-75.

8. Sarin SK, Kumar M, Lau GK, et al. Asian-Pacific clinical practice guidelines on the management of hepatitis B: a 2015 update. Hepatol Int 2016;10:1-98.

9. Saleh HA, Abu-Rashed AH. Liver biopsy remains the gold standard for evaluation of chronic hepatitis and fibrosis. $J$ Gastrointestin Liver Dis 2007; 16 .

10. Intraobserver and interobserver variations in liver biopsy interpretation in patients with chronic hepatitis $C$. the French METAVIR cooperative Study Group. Hepatology 1994;20:15-20.

11. Sanai FM, Keeffe EB. Liver biopsy for histological assessment: the case against. Saudi J Gastroenterol 2010;16:124-32.

12. Crossan C, Tsochatzis EA, Longworth L, et al. Cost-Effectiveness of non-invasive methods for assessment and monitoring of liver fibrosis and cirrhosis in patients with chronic liver disease: systematic review and economic evaluation. Health Technol Assess 2015;19:1-410.

13. Flores A, Asrani SK. Elastography in overweight and obese patients with chronic liver disease. Clin Gastroenterol Hepatol 2015;13:1510-2.

14. Attaullah $\mathrm{S}$, Khan S, Ali I. Hepatitis $\mathrm{C}$ virus genotypes in Pakistan: a systemic review. Virol J 2011;8.

15. Ahmad W, ljaz B, Javed FT, et al. A comparison of four fibrosis indexes in chronic HCV: development of new fibrosis-cirrhosis index (FCl). BMC Gastroenterol 2011;11:44.

16. Schmid $P$, Bregenzer $A$, Huber $M$, et al. Progression of liver fibrosis in HIV/HCV co-infection: assessment methods and liver biopsy. PLOS One 2015;10:e0138838.

17. European Association for Study of Liver. EASL clinical practice guidelines: management of hepatitis $\mathrm{C}$ virus infection. $J$ Hepatol 2014;60:392-420.

18. European Association for Study of Liver, Asociacion Latinoamericana para el Estudio del Higado. EASL-ALEH clinical practice guidelines: non-invasive tests for evaluation of liver disease severity and prognosis. J Hepatol 2015;63:237-64.

19. Fallatah HI. Noninvasive biomarkers of liver fibrosis: an overview. Adv Hepatol 2014;2014:1-15.

20. Ahmed Z, Ren J, Gonzalez A, et al. Universal index for cirrhosis (UIC index): the development and validation of a novel index to predict advanced liver disease. Hepat Med 2018;10:133-8.

21. Boursier J, de Ledinghen V, Zarski J-P, et al. Comparison of eight diagnostic algorithms for liver fibrosis in hepatitis C: new algorithms are more precise and entirely noninvasive. Hepatology 2012;55:58-67.

22. Zarski JP, Sturm N, Guechot J, et al. Comparison of nine blood tests and transient elastography for liver fibrosis in chronic hepatitis $\mathrm{C}$ : the ANRS HCEP-23 study. J He-patol 2012;56:55-62.

23. Afify SM, Tabll A, Nawara HM, et al. Five fibrosis biomarkers together with serum ferritin level to diagnose liver fibrosis and cirrhosis. Clin Lab 2018;64:1685-93.

24. Hussain Aet al. Role of ischemic heart disease (IHD), cerebral vascular accidents (CVA), dietary pattern and body mass index (BMI) in developing secondary systemic hypertension. Indo Am J P Sci 2018;05.

25. Hussain A, Noor HT, Nabi U. Evaluation of role of increased perceived stress score and body mass index in causing secondary systemic hypertension in patients of hepatitis C. J Cardiovasc Dis Diagn 2018;6.

26. Can we replace transient elastography i.e. fibro scan with cheap biomarkers? A cross sectional study at Lahore General Hospital, Lahore. Indo Am J P Sci 2019;06.

27. Hussain A, Khalid I, Fatima R, et al. Relationship of derangements in various blood indices and coagulopathy with liver stiffness index (Lsi) in various stages of fibrosis in hepatitis C. Indo Am J P Sci 2019;06. 